Tourón, J. (2020). Las Altas Capacidades en el sistema educativo español: reflexiones sobre el concepto y la identificación. Revista de Investigación Educativa, 38(1), 15-32.

DOI: http://dx.doi.org/10.6018/rie.38.1.396781

\title{
Las Altas Capacidades en el sistema educativo español: reflexiones sobre el concepto y la identificación
}

\author{
Gifted Students in the Spanish Education System: \\ Reflections on Conceptualization and Identification Issues
}

\author{
Javier Tourón \\ Catedrático de Métodos de Investigación y Diagnóstico en Educación \\ Universidad Internacional de La Rioja
}

\begin{abstract}
Resumen
El presente trabajo recoge una apretada síntesis de algunos de los problemas conceptuales que están vinculados con el desarrollo y la atención a los estudiantes con altas capacidades en el sistema educativo español, preferentemente no universitario. Así, parece esencial centrarse en el enfoque teórico de los modelos preeminentes en la literatura científica más relevante que permitan arribar a una mínima conceptualización operativa de los conceptos de capacidad y talento. Por otra parte, se analizan los problemas derivados de la identificación y su evidente déficit en las escuelas públicas y privadas. Algunas consideraciones a modelos relevantes de identificación basados en las medidas "out of level" son objeto de atención particular por su importancia metodológica y práctica, y por haber sido implantado por el autor durante años en España.

Palabras clave: Alta capacidad; dotación; talento; aceleración educativa; enriquecimiento.
\end{abstract}

Correspondencia: Javier Tourón, javier.touron@unir.net Vicerrector de Innovación y Desarrollo Educativo. Universidad Internacional de la Rioja. c/ Av. de la Paz, 137. 26006 Logroño.

Agradecimientos: Esta investigación fue financiada por el Ministerio de Economía, Industria y Competitividad de España, RTI2018-094212-B-I00: (CIBER-AACC). This research was funded by Spanish Ministry of Economy, Industry and Competitiveness, RTI2018-094212-B-I00: (CIBER-AACC). 


\begin{abstract}
This paper presents a synthesis of some of the conceptual problems related with the development of services offered to gifted students in the Spanish education system, mainly in the nonuniversity levels. Thus, it seems essential to focus on the theoretical approach of the preeminent models in the most relevant scientific literature that allow us to get a minimum operational conceptualization of the concepts of ability and talent. On the other hand, the problems derived from the lacking identification processes in public and private schools are analyzed. Some considerations to relevant identification models based on the "out of level" measures are payed particular attention for their methodological and practical importance and for having been implemented by the author for years in Spain.
\end{abstract}

Keywords: High ability; giftedness; talent; acceleration; enrichment.

\title{
Introducción
}

Hay pocos temas en el ámbito educativo que hayan sido objeto de tanta atención por parte de estudiosos e investigadores como el de los alumnos de alta capacidad. Tradicionalmente este ámbito era referencia obligada dentro de la Pedagogía Diferencial, de la que tenemos exponentes destacados en nuestra área. El hoy departamento MIDE de la Universidad Complutense, lo fue originalmente de Pedagogía Experimental y Diferencial, a cuyo frente estuvo el profesor García Hoz, principal introductor de ambas áreas en España y proponente de la Educación Personalizada (García Hoz, 1975; Vázquez, 1981). Su sucesor al frente de dicho departamento, el profesor Orden Hoz, también cultivó ampliamente el ámbito de la Pedagogía Diferencial, con destacados trabajos sobre el agrupamiento escolar y otros ámbitos del estudio diferencial de la educación, como puede verse en la obra "Elogio a la Pedagogía Científica" (Cf. Castro Morena, 2012; Orden Hoz, 1975).

A lo largo del tiempo se han ido publicando trabajos diversos sobre Pedagogía Diferencial que incluían una referencia más o menos extensa al tema que nos ocupará en estas páginas (ver p. e. Jiménez y Galán, 2011; Jiménez, 1998).

Nos referimos aquí la Pedagogía Diferencial como pedagogía de las diferencias individuales, un área de investigación que, lejos de formar parte de la historia de la Pedagogía, debe tener más importancia hoy, pues las tecnologías digitales a nuestro alcance hacen más viable la puesta en práctica de procesos de diferenciación e individualización, de personalización en suma, que en el pasado se fueron abandonando -aun habiendo mostrado su eficacia- por el alto coste de recursos que requerían.

López, Tourón y Galán, (1990, p. 86) señalaban que:

La Pedagogía Diferencial que proponemos, toma como punto de arranque algo que es elemental, la referencia al individuo, a la persona, como sujeto de la educación. No parece necesario a estas alturas tener que señalar que las supuestas homogeneidades grupales son un mito. Los alumnos son diferentes, con características diversas y con ritmos de aprendizaje diferentes. Esto obliga a pensar en cómo adecuar la educación a tales diferencias, que no es otra cosa que individualizar [personalizar] la educación y, por tanto, la ense- 
ñanza. Lo que proponemos es construir una Pedagogía Diferencial que tome como punto de mira la adecuación a las diferencias humanas individuales.

Como ocurrió con otras disciplinas y ámbitos que estaban albergados dentro de la Pedagogía Diferencial, este campo de estudio ha ido adquiriendo tímidamente una cierta relevancia e independencia, sin que esté claro que se haya consolidado en nuestro país como un ámbito de estudio autónomo; cosa bien distinta de lo que ha ocurrido en Europa (Cf. Mönks y Pfluger, 2005) y desde luego en los EE.UU. (y países anglosajones también) donde existe una extraordinaria comunidad de investigadores con una producción muy notable desde hace más de un siglo (Cf. NAGC, 2019).

Pocas variables hay tan relevantes como la capacidad, las aptitudes, para diferenciar la educación de las personas, no discriminándolas, sino potenciando el desarrollo óptimo de su potencial. Precisamente la investigación señala, con evidencias palmarias, que cuando se personaliza el aprendizaje el rendimiento de todos los escolares mejora, haciendo verdadero el aforismo inglés: "a rasing tide lifts all boats".

Apuntado pues el ámbito en el que nuestro tema se enmarca es momento de comenzar su desarrollo. En las próximas páginas abordaré, sucintamente, por razones obvias de espacio, dos cuestiones: a) la conceptualización; b) la identificación.

\section{Alta capacidad y talento: precisiones conceptuales}

El problema conceptual que se da en este campo de estudio es mayúsculo y tiene consecuencias indeseables para la identificación y la intervención, lo que no es exclusivo de nuestro país. En cierta medida, ocurre en el ámbito anglosajón dentro de la comunidad educativa, no investigadora. Una primera problemática se deriva de la equiparación de términos como gifted, que quiere decir dotado, y hacerlo equivaler a superdotado (su correlato en ingles sería supergifted, que no existe), y este último ligarlo a la obtención de un valor de CI, generalmente 130, en alguno de los tests al uso. El problema central aquí es que la etiqueta se hace equivaler a un "estado del ser", o a un rasgo o cromosoma de oro (Cf. Renzulli y Reis, 2018), de manera que unas personas lo poseen y otras no. Se convierte de este modo el hecho de ser gifted en una variable dicotómica, cuyas categorías se establecen arbitrariamente a partir de un punto de corte. Con rotunda claridad señalan estos mismos autores:

El término 'dotado' [gifted en el original] significa que uno es excepcional en algo y preferimos usar la palabra "dotado" como adjetivo (por ejemplo, él o ella es un/a pianista, escritor/a, etc. dotado/a), en vez de como un sustantivo (ella es dotada). También preferimos hablar de comportamientos dotados (adjetivo) en lugar de usar los 'dotados' para representar un estado de ser (p.185).

Hay varias razones por las que hablar de superdotado (o superdotación) es inadecuado, a pesar de que mucha legislación se empeña en ello de manera evidente. Destaco, resumidamente algunas.

Hay que entender que no se trata de un atributo, o condición natural, de la que unos gozan y otros no. Por ello, no puede plasmarse en una puntuación de CI, y si 
bien esta puede tener cierta utilidad, es de escaso o nulo valor para organizar la intervención educativa. Todos los modelos actuales, como se verá, enfatizan la importancia del desarrollo a lo largo de la vida de la persona, estableciendo la importancia del impacto del entorno en dicho desarrollo. En este sentido se puede afirmar que todos estamos en proceso de ser.

Así visto, la capacidad se convierte en la materia prima del talento. Señala el eventual potencial a desarrollar por la persona, cuando esas capacidades se apliquen a cualquier campo relevante de la actividad humana. Algo que no ocurre de manera espontánea.

Por ello, parece sensato entender que esta conceptualización nos ha de poner en primer plano la necesidad de identificar los potenciales diversos de las personas para ayudarlas a convertirlos en talentos o competencias desarrolladas, en rendimiento (Cf. Tourón, 2012).

Más aún, como señala Pfeiffer (2017), el término gifted es un constructo social, una denominación que utilizamos para referirnos a un grupo heterogéneo de personas que se caracterizan por tener una alta capacidad, un alto rendimiento o potencial para rendir.

\section{Breves pincelas históricas: de la dotación al talento}

No tiene objeto en esta síntesis abordar de modo prolijo la historia y la evolución de las diferentes teorías aparecidas a lo largo de los años, pero si cabe señalar un cierto itinerario conceptual para hacer unas mínimas referencias a los modelos preponderantes hoy en día y que gozan de la aceptación y el consenso de la comunidad científica. Las fuentes que se citan ayudarán al lector interesado a profundizar en lo que aquí apenas se esboza.

Los primeros estudios relativos a las altas capacidades nacen paralelas al estudio de la inteligencia, lo que es comprensible pues entre los rasgos que identifican a las personas con alta capacidad sobresale su inteligencia elevada. Exponentes primigenios (sin retroceder, por ejemplo, al Examen de ingenios de Huarte de Sanjuan) son Galton (1822-1911) y sus estudios recogidos en Hereditary Genius (1869) y English Men of Science (1874). Los trabajos de Alfred Binet y la medida de la inteligencia estableciendo el concepto de edad mental; o los relevantes estudios de Lewis Terman, una figura de obligada referencia en este ámbito. Su estudio longitudinal sobre 1528 niños realizado en la Universidad de Stanford, utilizando como criterio de selección poseer un CI de 140 o superior en la prueba Stanford-Binet (adaptación americana de la prueba desarrollada en Francia por Binet y Simon, publicada en 1905), se publicó en varios volúmenes entre los años 1925 y 1959 (Genetic Studies of Genius, 1925-1959). Por su parte, Letta Hollingworth (1926) hizo contribuciones significativas para apoyar a los niños más capaces y optimizar su educación, desarrollar programas específicos, y comenzar a establecer las primeras diferencias entre los conceptos de dotación y talento. La diferencia entre estos conceptos fue perfilada años después por De Haan y Havinghurst (1957), como un anticipo de lo que más adelante se convertirá en un aspecto esencial dentro del estudio de las personas de alta capacidad.

Un paso adelante dentro de este paralelismo entre el estudio de la inteligencia y las altas capacidades lo da Guilford en 1967 cuando propone su concepto de inteligencia 
humana, alejándose de concepciones unidas al CI (Structure of Intellect). Plantea este autor, como es conocido, 150 factores articulados en tres dimensiones que denomina: operaciones (cómo pensamos), contenidos (qué pensamos), y productos (los resultados) conseguidos al aplicar una determinada operación a un contenido dado.

Un hito destacado en esta trayectoria es el informe Marland (1972), de la oficina federal de Educación de los EE.UU. En el mismo se aporta una primera definición de dotación y talento [gifted and talented] ${ }^{11}$. Los factores no intelectivos, sin embargo, quedan bastante al margen en la definición. Cuestión que otras aportaciones posteriores corregirán.

Richert, Alvino y McDonnel (1982) ${ }^{2}$ hicieron un estudio sobre las posturas adoptadas en los Estados Unidos para conceptualizar e identificar a los más capaces, que habría de tener una gran repercusión. Los autores concluyen que, hasta ese momento, las definiciones predominantes que condicionan la identificación y la educación de los sujetos de alta capacidad se pueden agrupar en cinco categorías: a) las ligadas a la capacidad intelectual excepcional: de enfoque eminentemente psicométrico y frecuentemente asociada a una puntuación de CI en torno a dos desviaciones típicas por encima de la media; b) centradas en las aptitudes intelectuales diferenciales: que pretendían evitar la consideración de dos categorías, como las anteriores: los "dotados" y los "no dotados" (you have it or you don't; gifted or not gifted). La aportación más destacada es la de Guilford a la que ya hemos aludido; c) las que consideran el papel de la creatividad; d) las relativas a los talentos múltiples: que permiten una concepción mucho más amplia y compleja de la dotación; la definición Marland, también conocida como la definición federal. Los autores concluyen que rara vez se pasaba de los dos primeros enfoques en la práctica educativa de los Estados.

Es posible observar desde los años ochenta un cambio de enfoque, un nuevo modo de entender la dotación que puede percibirse en algunos aspectos. La mayoría de los supuestos coinciden en afirmar que: 1) dotación se muestra en la producción de un trabajo valorado socialmente, 2) durante la infancia sólo puede identificarse una dotación potencial, y 3) la estimación del potencial para la productividad adulta es factible (Jackson y Butterfield, 1986). Sin embargo, persisten las discrepancias respecto a la elección de aquellos criterios que constituyen la clave para la identificación de la dotación en la infancia.

1 Niños dotados y con talento son aquellos que han sido identificados por profesionales cualificados, en virtud de sus habilidades destacadas y por su alto rendimiento. Estos niños requieren programas de educación diferenciada y servicios distintos de los proporcionados habitualmente en un centro ordinario para que puedan aportar su contribución a sí mismos y a la sociedad. Los niños capaces de alto rendimiento incluyen aquellos con rendimiento demostrado o capacidad potencial en cualquiera de las áreas siguientes, bien en una o en varias: 1) capacidad intelectual general, 2) aptitud académica específica, 3) pensamiento creativo o productivo, 4) capacidad de liderazgo, 5) artes visuales y manipulativas, 6) capacidad psicomotora. Es presumible que la utilización de estos criterios para la identificación de los sujetos dotados y con talento incluirá un mínimo del 3-5\% de la población escolar (Marland, 1972).

2 Esta tarea la sigue llevando a cabo la NAGC que publica informes periódicos sobre el Estado de la Nación en relación con los alumnos identificados, las ayudas estatales, los programas disponibles, etc. Puede verse un ejemplo aquí: http://www.nagc.org/resources-publications/gifted-state/2014-2015-statestates-gifted-education 
El concepto, en cualquier caso, sufre un cambio de enfoque que se hace más patente a mediados de los ochenta y que sintetizan muy bien las aportaciones de Treffinger (1991) y Feldman (1992). Los aspectos principales de este cambio que se menciona se resumen en la tabla 1, que adaptamos de Tourón y cols. (1998).

Tabla 1

Elementos diferenciales del paradigma tradicional y actual en las dimensiones que se señalan respecto a las altas capacidades.

\begin{tabular}{|c|c|c|}
\hline Aspecto & Paradigma tradicional & Paradigma actual \\
\hline \multirow{3}{*}{ CONCEPTO } & $\begin{array}{l}\text { La dotación es igual a un elevado } \\
\text { CI. }\end{array}$ & $\begin{array}{l}\text { La dotación es multifacética; se } \\
\text { manifiesta de diferentes formas. }\end{array}$ \\
\hline & $\begin{array}{l}\text { La dotación es un rasgo simple, } \\
\text { innato y no cambiante. }\end{array}$ & $\begin{array}{l}\text { La dotación es multidimensional } \\
\text { sujeta a desarrollo y cambio. }\end{array}$ \\
\hline & $\begin{array}{l}\text { Algo estrictamente cuantitativo y } \\
\text { psicométrico. }\end{array}$ & $\begin{array}{l}\text { Algo cualitativo, no meramente } \\
\text { cuantitativo. }\end{array}$ \\
\hline \multirow[b]{2}{*}{ IDENTIFICACIÓN } & Basada en los tests. & Basada en el rendimiento. \\
\hline & $\begin{array}{l}\text { Exclusiva, busca sólo los “auténti- } \\
\text { cos" estudiantes dotados. }\end{array}$ & $\begin{array}{l}\text { Inclusiva, intenta promover el } \\
\text { potencial de los estudiantes. } \\
\text { Diagnóstica, destinada a mejorar } \\
\text { la planificación instructiva. }\end{array}$ \\
\hline \multirow{5}{*}{$\begin{array}{l}\text { RESPUESTA } \\
\text { EDUCATIVA }\end{array}$} & $\begin{array}{l}\text { - La dotación se expresa sin inter- } \\
\text { vención especial. }\end{array}$ & \multirow{3}{*}{$\begin{array}{l}\text { - La dotación precisa interven- } \\
\text { ción específica. } \\
\text { - Programas para la dotación o } \\
\text { estimulación de las conductas } \\
\text { dotadas (gifted behaviors) }\end{array}$} \\
\hline & - Programas únicos para todos. & \\
\hline & $\begin{array}{l}\text { - Currículo prefijado, contenidos } \\
\text { fijados }\end{array}$ & \\
\hline & $\begin{array}{l}\text { - Dirección centrada en el profe- } \\
\text { sor. }\end{array}$ & $\begin{array}{l}\text { - Opciones, flexibilidad en la elec- } \\
\text { ción y la duración. }\end{array}$ \\
\hline & $\begin{array}{l}\text { - Derivada de inferencias obteni- } \\
\text { das a partir de otros alumnos } \\
\text { dotados o con talento. }\end{array}$ & $\begin{array}{l}\text { - Basada en las características } \\
\text { actuales de los estudiantes. }\end{array}$ \\
\hline
\end{tabular}

Nota: el término dotación en este contexto es sinónimo de capacidades o altas capacidades (o aptitudes).

Se han propuesto muchas teorías y clasificaciones de los diversos modelos existentes (ver p. e. Tourón, Peralta y Repáraz, 1998; Pfeiffer, 2017). El propio Pfeiffer (2018) en un trabajo reciente ${ }^{3}$ propone una clasificación que permite englobar la mayor parte de los modelos: [1] el psicométrico, [2] el desarrollo del talento, [3] el rendimiento experto y [4] las inteligencias múltiples. Hay otras clasificaciones como las que pueden encontrarse en

3 Puede encontrarse traducido al castellano, con autorización del autor en: https://www.javiertouron. es/alta-capacidad-y-desarrollo-del-talento-en-ninos-y-jovenes-concluyendo/ 
Sternberg y Davidson (2005) o en los Handbooks clásicos sobre el tema (Cf. p. e. Heller, Mönks, Subotnik y Sternberg, 2000; Colangelo y Davis, 2002; Pfeiffer, 2018; Shavinina, 2009).

Es posible señalar que la evolución histórica del concepto nos ha llevado de ver a las personas de alta capacidad ligadas a una inteligencia alta ("ser capaz o muy capaz" es tener un CI alto). No obstante, no existe procedimiento posible, a través de una puntuación basada en un test de inteligencia, que permita distinguir a los alumnos de gifted de los non-gifted. Y ello, básicamente, porque la capacidad es una cuestión de grado, no una variable dicotómica. Actualmente hemos pasado a entender la inteligencia como una realidad poliédrica (se puede ser de alta capacidad de modos diversos), ello ligado a los modelos factoriales de la inteligencia, para posteriormente considerar la importancia del medio, del entorno y de las variables no intelectivas, maleables, de corte psicosocial, de manera que, como señalan Olszewski-Kubilius, Subotnik y Worrel (2015, p. 41):

La alta capacidad [giftedness] es la manifestación del rendimiento que se encuentra claramente en el extremo superior de la distribución en un dominio de talento específico, incluso en relación con otros individuos de alto nivel de funcionamiento en ese dominio. Más aún, la alta capacidad puede verse como un proceso de desarrollo en el que, en las primeras etapas, el potencial es la variable clave; en etapas posteriores, el rendimiento es la medida de la alta capacidad; y en los talentos completamente desarrollados, la eminencia es la característica que hace acreedor a esta denominación. Tanto las variables cognitivas como las psicosociales juegan un papel esencial en la manifestación de la alta capacidad en cualquiera de los estadios del desarrollo, son maleables y necesitan ser deliberadamente cultivadas.

Este enfoque, claramente evolutivo, es el adoptado por Pfeiffer en su modelo tripartito ya citado, o el propio Renzulli (Cf. Renzulli y Reiss, 2018; Renzulli y Geasser, 2015). Precisamente este autor señala, refiriéndose a su modelo:

El modelo de los tres anillos se basa en la interacción y solapamiento de tres conjuntos de rasgos que, a su vez crearán, las condiciones para la aparición y manifestación de lo que se denominan conductas dotadas (gifted behaviors). Este enfoque no concibe, por tanto, la alta capacidad intelectual como una característica absoluta y estable (esto es, 'que se tenga o que no se tenga'). Se concibe más bien como un conjunto de conductas desarrollables dentro del marco de la resolución de problemas. Distintos tipos y grados de conductas dotadas podrán ser, por tanto, desarrolladas y, en definitiva, reconocibles en ciertas personas, en ciertos momentos y bajo ciertas circunstancias (Renzulli y Geasser, 2015, p. 109).

En un trabajo posterior, Renzulli y Reis, $\left(2018^{4}\right)$ enfatizan claramente el carácter evolutivo y multidimensional de las capacidades, distinguen además entre las capacidades

4 Una versión en castellano puede encontrarse en: https://www.javiertouron.es/modelo-de-los-tresanillos-vision-global/ 
académicas y las productivo-creativas, distinción que ya había planteado Renzulli en 1978 cuando propuso su modelo de los tres anillos por primera vez ${ }^{5}$.

Una última referencia obligada es la de Gagné (2015, pp. 15-16) que sintetiza de modo global su modelo integrador de dotación y talento en el que distingue estos dos conceptos, de manera que

la dotación designa la posesión y el uso de capacidades naturales sobresalientes sin entrenamiento y espontáneamente expresadas, también llamadas aptitudes (o dones), en al menos un dominio de capacidad, en un grado que coloca al individuo, por lo menos, entre el 10\% superior de los compañeros de su edad. Talento designa el dominio excepcional de competencias desarrolladas sistemáticamente (conocimientos y capacidades) en, al menos, un campo de la actividad humana, en un grado que coloca al individuo, por lo menos, entre el 10\% superior de "compañeros" (aquellos que han acumulado una cantidad similar de aprendizaje en el mismo tiempo).

Un buen número de los modelos más importantes sobre este asunto se encuentran recogidos en la monografía de la Revista de Educación 368 de 2015 , y en las obras citadas anteriormente, en particular los Handbooks mencionados, de manera especial el de Sternberg y Davidson (2005) dedicado monográficamente a las Conceptions of Giftedness.

Para sintetizar este apartado, podemos resumir algunos aspectos clave de los diversos modelos, en particular los más actuales y con mayor respaldo de la investigación y la comunidad científica (Renzulli, Pfeiffer, Gagné, Subotnik, Olszewski-Kubilius y Worrel), siguiendo lo que ya señalamos en trabajos anteriores (p. e. Tourón, Peralta y Repáraz, 1998; Reyero y Tourón, 2000, entre otros).

Ante la posible conclusión precipitada, de que los autores no están de acuerdo, se debe señalar que, aunque los modelos son diferentes en muchos aspectos, tienen una importante base común y no deben considerarse excluyentes.

Las capacidades cognitivas constituyen una parte esencial de la dotación, particularmente cuando hablamos de su dimensión académica. No obstante, hay un gran número de factores tanto innatos como ambientales (sociales, culturales y educativos) que juegan un papel determinante en el desarrollo de esta. Por ello, el estudio de la alta capacidad y la inteligencia han corrido paralelos durante buena parte del siglo pasado (Cf. para más detalle Reyero y Tourón, 2000).

Es relevante tener en cuenta también que la dotación no es sólo un fenómeno cognitivo que pueda ser medido a través de instrumentos tradicionales como los tests de CI (una puntuación alta de CI, puede ser una condición necesaria, aunque no suficiente si proponemos un concepto de dotación más amplio que el concepto centrado en la dotación intelectual o en los talentos académicos). Es importante tener en cuenta otras dimensiones no cognitivas como la creatividad, la motivación, el afecto y otras variables de la personalidad, el entorno social y familiar que, junto las circunstancias vitales de

5 Ver, para más detalle: https://gseuphsdlibrary.files.wordpress.com/2013/03/what-makes-giftedness.pdf

6 Accesible en abierto. Se puede recuperar desde: http://www.educacionyfp.gob.es/revista-de-educacion/numeros-revista-educacion/numeros-anteriores/2015/368.html 
las personas son factores tan determinantes como la cognición. La compleja interacción de estos constructos hará, en el mejor de los casos, que el potencial latente emerja.

En cualquier caso, debe desterrarse la idea de que la dotación es un rasgo simple, innato, que no cambia, y pasar a considerarla como una realidad multidimensional sujeta a desarrollo y cambio. El foco de atención se ha desplazado, según Treffinger y Feldhusen (1996), a las aptitudes específicas y las capacidades que surgen en áreas particulares de talento.

Por todo ello, se puede concluir que la dotación no es un rasgo unidimensional, sino un fenómeno multidimensional que se manifiesta de distintas formas y en diferentes niveles según las personas, las circunstancias y el momento de que se trate. No es, por tanto, estático, si no que evoluciona a lo largo del desarrollo vital de la persona como resultado de la interacción entre las capacidades innatas y el apoyo apropiado.

Otro aspecto importante, como señalamos en otro momento (Cf. Tourón y cols. 1998; Reyero y Tourón, 2000), es la necesidad de diferenciar entre la dotación y el talento, aunque en la mayoría de las personas capacidades y talentos coexisten. El término dotado corresponde a una capacidad claramente por encima de la media en uno o más dominios de la aptitud humana, en relación con su grupo de iguales (en edad o en capacidad). El término talento se concibe como una competencia especial para determinadas áreas de la actividad humana. Es el resultado de la conversión de las capacidades naturales en capacidades sistemáticamente desarrolladas, que podemos llamar competencias (Gagné, 2015).

Lo relevante de la conceptualización, que apenas hemos incoado en las páginas anteriores, es entender que no estamos hablando, por volver al principio, de un asunto de ser o no ser o de un rasgo con el que unos nacen y otros no, sino de una condición de grado que está sujeta a desarrollo. Como hemos señalado en alguna ocasión, entre la capacidad o aptitud y el talento o competencia, están los catalizadores ambientales e intrapersonales, en nomenclatura de Gagné. Dicho en otros términos, entre las capacidades naturales y las sistemáticamente desarrolladas está la educación, la intervención educativa si se quiere, de manera que a la capacidad han de asociarse un buen número de variables psicosociales, maleables, en ocasiones denominadas de modo genérico soft skills (o core skills), que han de ser responsables, junto con la práctica deliberada, el trabajo y el esfuerzo, del florecimiento del talento. Así lo señalaba Pfeiffer (2012),

Además de la capacidad intelectual general, las capacidades específicas y un buen número de factores no intelectivos, contribuyen de manera decisiva a configurar una trayectoria de éxito en los jóvenes más capaces. La práctica deliberada contribuye a predecir el rendimiento experto en muy diversos dominios. (...) La propia experiencia confirma que el trabajo duro, la recompensa diferida, y la autodisciplina también son críticamente importantes, incluso entre los estudiantes más capaces (p. 6).

O como señalaron hace ya un tiempo Treffinger y Feldhusen (1996), los talentos emergen y crecen evolutivamente, y para algunos no llegan a emerger porque no se produce una adecuada estimulación en la escuela y la familia. Es imperativo que 
todos los que trabajan con jóvenes vean los talentos y potencialidades como algo educable y emergente, y no como algo fijo e inmutable.

Luego si el talento no se expresa de manera espontánea, sino como una combinación de capacidad, entorno y trabajo, la identificación se vuelve imprescindible si pretendemos que los más capaces conviertan su potencial en rendimiento en el ámbito que fuere.

\section{Identificación de los alumnos de alta capacidad: un problema acuciante}

¿Es posible desarrollar lo que apenas son dudosas potencialidades y convertirlas en rendimiento sin una intervención educativa específica? Si la dotación fuera una cuestión de ser o no ser, de rasgo, la identificación no sería muy necesaria, ya que las personas vendrían determinadas en función de sus condiciones de nacimiento, algo que carece de sentido incluso plantear. Si el desarrollo del talento fuera espontáneo, o si la escuela en su intervención ordinaria ofreciese los mecanismos adecuados para desarrollar el potencial de todos los alumnos, la identificación sería innecesaria.

Dicho de otro modo,

si la escuela fuese verdaderamente adaptativa y respondiese de modo individualizado [personalizado] a las necesidades de cada uno de los aprendices, la dotación no sería un problema educativo (...), sin embargo, es patente que la escuela y los programas que en ella se desarrollan dista mucho de adaptarse a las necesidades de todos los educandos. (Tourón y Reyero, 2002, p. 311). El que alumnos diferentes sometidos al efecto de un mismo programa educativo o currículo, no obtengan resultados diferentes, nos pone en la pista de que las diferencias constituyen un problema educativo que acaba uniformando a los que son diversos.

Es un error, casi universalizado, de los sistemas educativos el ligar 'desarrollo educativo' y 'edad', para organizar los grupos de alumnos en función de la última, olvidando las diferencias que se producen en el desarrollo de las personas.

¿Qué es un niño precoz si no?, ¿qué decir de las diferencias en el ritmo de aprendizaje? Los sistemas educativos tienen que promover la excelencia, no asegurar mínimos. Esto quiere decir que es preciso establecer procedimientos de búsqueda sistemáticos y regulares que, vinculados de modo adecuado a programas educativos diversos, permitan identificar a todos aquellos posibles alumnos que por sus características personales no van a ser adecuadamente estimulados por los programas regulares. Esta política nos llevaría a una promoción activa y decidida de todo tipo de talentos (Tourón y Reyero, 2002, p. 312).

De acuerdo con cifras ofrecidas por el propio Ministerio de Educación, es posible comprobar que, dependiendo de las regiones, más del 90\% de los alumnos más capaces están sin ni siquiera detectar. Si esto es así, poco podemos esperar del desarrollo de su talento de acuerdo con lo que va dicho. 


\section{Características de un proceso de identificación}

La literatura sobre identificación y las características que debe reunir este proceso es abundantísima (p. e. Clark, 1992; Feldhusen y Baska, 1985; Feldhusen y Jarwan, 1993; Hagen, 1980; Piirto, 1999; Tannenbaum, 1983; Pfeiffer, 2015; Renzulli y Geasser, 2015; Lubinski; Benbow, y Kell, 2014, entre otros muchos). Las propuestas son complementarias y deben ser coherentes con dos aspectos: el concepto o enfoque de la dotación que se adopte y las características de los programas que se ofrezcan para su desarrollo. Es particularmente interesante el trabajo de Renzulli y Geasser (2015) sobre el establecimiento de un sistema multicriterial de identificación, en el que se incluye su conocido modelo de puerta giratoria, sobre el que ya escribieron Tourón y cols. (1998). Aunque es quizá, el Talent Search, al que nos referiremos enseguida, el modelo más difundido en la literatura y práctica educativa, aunque muy ignorado en nuestro entorno.

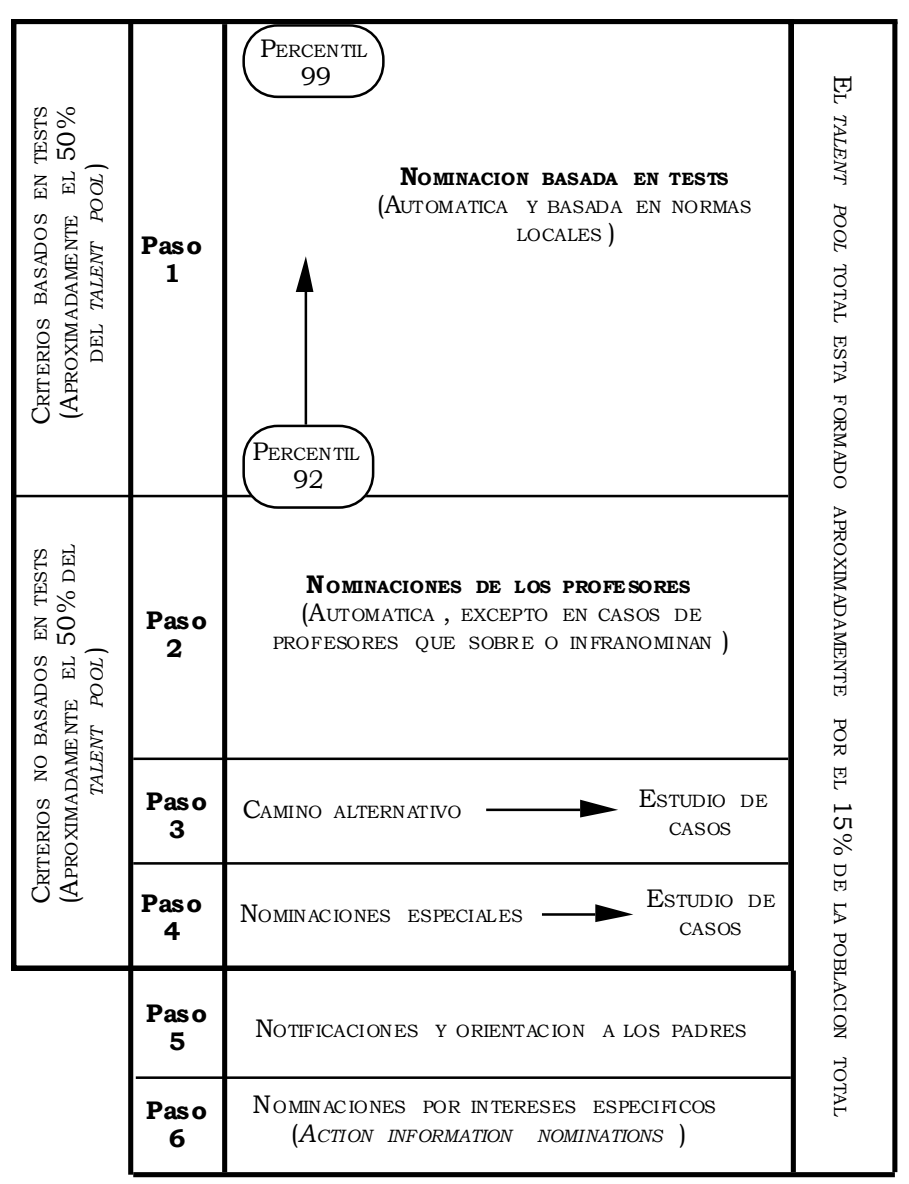

Figura 1. Revolving Door Identification Model de Renzulli (tomado de Tourón y cols. 1998) 
También es muy recomendable para el lector interesado el libro, traducido al castellano, de Pfeiffer (2017), dedicado a la identificación y evaluación del alumnado con alta capacidad. Sin duda la referencia actualmente más autorizada en nuestra lengua.

El National Report on Identification: Assessment and Recommendations for Comprehensive Identification of Gifted and Talented Youth (Richert, S., Alvino, J., y McDonnel, R., 1982) resume las características que debe reunir cualquier proceso de identificación del siguiente modo: los intereses de todos los alumnos deben ser prioridad de todo el proceso (advocacy); los procedimientos que se empleen han de basarse en las mejores evidencias de investigación disponibles (defensibility); se debe buscar la equidad para evitar que ningún alumno sea excluido equivocadamente o por razones espurias ajenas al proceso (equity); se recomienda utilizar un enfoque amplio (pluralism) y, finalmente, debe procurarse que el mayor número de alumnos sean identificados y atendidos (comprehensiveness). Puede ser interesante para el lector revisar también el trabajo posterior de Richert (1991) sobre este particular.

Si hubiésemos de resumir, podríamos decir que se han propuesto dos grandes modelos de identificación, el de puerta giratoria, que es el modelo que enlaza con la propuesta de los tres anillos de Renzulli y el Talent Search. Sobre el primero, que aquí no trataremos por razones de espacio (lo representamos en la figura 1 a título informativo), pueden consultarse Tourón y cols. (1998), Tourón y Reyero (2002) o los recientes trabajos de su autor, en particular Renzulli y Geasser (2015).

\section{El Talent Search? : la importancia del out of level}

Este modelo que, sin duda, es el que tiene mayor investigación tras de sí, fue promovido por Stanley a finales de los años 60 (Cf. Stanley, 2005). Al principio sus ideas fueron consideradas algo descabelladas, hoy en día son cientos de miles los alumnos identificados con arreglo a este modelo cada año y los Talent Search han venido a representar una auténtica revolución en la educación de los alumnos más capaces (Cf. Colangelo, Assouline y Gross, 2004).

El primer Talent Search se llevó a cabo en 1972 con 450 alumnos del área metropolitana de Baltimore ${ }^{8}$. Se pretendía identificar a jóvenes "que razonaban extraordinariamente bien en Matemáticas" a una edad temprana y que, por tanto, precisaban de una planificación educativa que atendiese a su competencia antes que a su edad. El interés inicial por la dimensión cuantitativo-matemática se amplió al área verbal. La capacidad de predicción de ambas áreas para el desarrollo del potencial académico de los jóvenes se ha puesto de manifiesto en múltiples estudios (ver p. e. Benbow, 1992).

El problema educativo de los alumnos más capaces en la escuela se hace patente cuando se aprecia que estudiantes que son distintos en sus capacidades, velocidad de aprendizaje, motivación e intereses, etc., no obtienen resultados distintos a los de sus compañeros de edad cuando están bajo la influencia de un mismo currículo, igual para

7 Puede accederse a una amplia selección de entradas del blog del autor, relativas al Talent Search y sus principios, desde este enlace: https://www.javiertouron.es/?s=Talent+Search. También desde https:// www.javiertouron.es/javier-touron/ puede accederse a una amplia bibliografía del autor sobre el tema.

8 Pueden encontrarse descripciones detalladas en castellano en Tourón (2004) o en Reyero y Tourón (2003). 
todos, desarrollado a la misma velocidad y con el mismo nivel de profundidad y amplitud para todos. Se hace patente así la necesidad de una educación diferenciada que les permita optimizar el desarrollo de su potencial. O recibir un nivel de reto que está a la altura de su potencial. En caso contrario su potencial no se desarrollará óptimamente.

En la figura 2 se resume el proceso de identificación bajo los principios del Talent search, en particular respecto a la medida de las capacidades verbales y cuantitativas (Tourón, 2004). Los alumnos que son evaluados con tests generalmente curriculares (que miden lo que deben saber y saber hacer en cada materia y edad los estudiantes) (fase 'in level') y obtienen en los mismos puntuaciones que les sitúan en el 5\% superior respecto a sus compañeros de edad, pueden presentar entre ellos grandes diferencias de capacidad y dominio. Stanley conociendo de manera directa la gran capacidad para manejarse con currículos muy por encima de los que les corresponderían por edad a estos alumnos, lanzó la hipótesis de que también podrían hacerlo con tests desarrollados para alumnos mayores. Así, y aprovechando las facilidades de los sistemas de evaluación del sistema norteamericano, aplicó el SAT (Scholastic Assessment Test) a estos alumnos que comparados con sus compañeros rendían de manera sobresaliente.

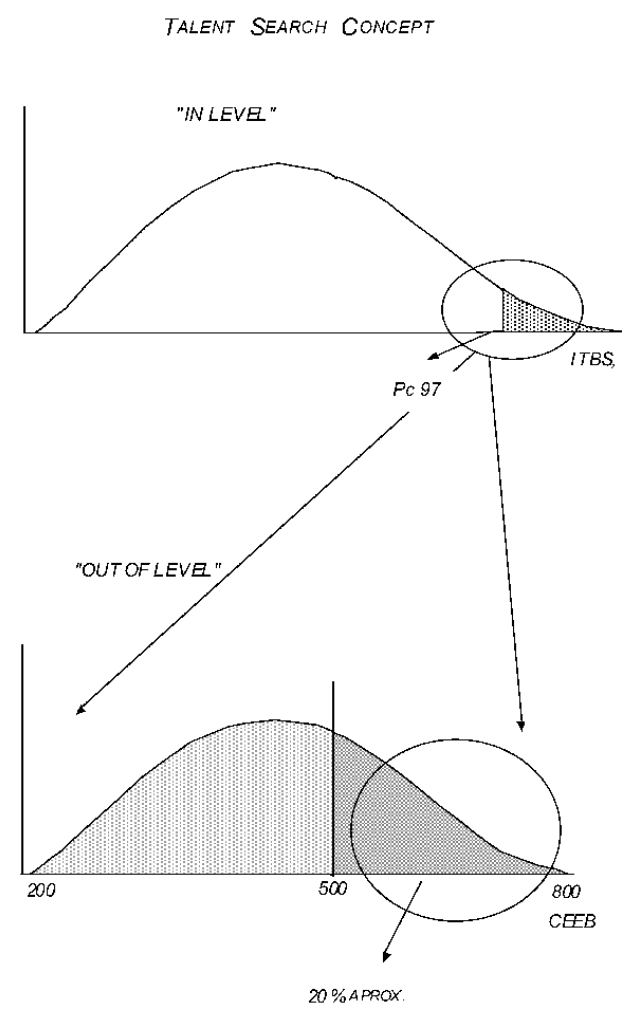

Figura 2. El modelo de identificación Talent Search original de Julian Stanley 
Así, se establece esta segunda fase, en la que los estudiantes son evaluados con tests como el mencionado SAT o el ACT (American College Testing). En síntesis, se ve que los alumnos que en la fase in level obtienen puntuaciones como se ha señalado, al menos en torno al 20\% de ellos igualan, o mejoran, las puntuaciones de los estudiantes que acceden a la universidad (varios años mayores que ellos). Esta pauta se viene repitiendo desde hace más de treinta años en todos los talent searches que se llevan a cabo. Resultados de la aplicación de este modelo en España pueden verse en Tourón; Tourón y Silvero (2005) o en Tourón y Tourón (2016).

No podemos tratar esta cuestión con extensión aquí, pero el lector puede consultar los trabajos de Barnett y Gilheany (1996), para una experiencia similar en Irlanda; Barnett y Corazza, (1993); Goldstein, Stocking y Godfrey, (1999); hay descripciones más o menos detallas en las obras de Stanley y Benbow, (1981, 1982, 1983, 1986), entre otras muchas. Los trabajos del autor citados también dan cuenta con cierto detalle del análisis del talent search y de su carácter universal o transcultural si se quiere decir así.

Podríamos preguntarnos ¿por qué es preciso hacer una medición fuera de nivel? La respuesta es simple: es preciso saber cuál es el grado de capacidad o talento de un alumno dado para poder planificar su itinerario de aprendizaje. Ordinariamente más capacidad implica más velocidad de aprendizaje. Esto es coherente con un enfoque evolutivo del desarrollo del talento, como se señaló al principio.

Así pues, debería considerarse la necesidad de re-evaluar a los alumnos que obtienen puntuaciones elevadas al ser comparados con otros estudiantes de su edad, para mitigar o eliminar el posible efecto de techo de las pruebas que se empleen. Será, además, el único modo de establecer las diferencias reales que existen también entre los más capaces y ofrecerles la posibilidad de tener un itinerario educativo que los lleve a realizar todo su potencial (ver Clynes, 2016). (Para un análisis detallado del 'out of level' en España ver Tourón y Tourón, 2016).

\section{Discusión y conclusiones}

La conceptualización de la alta capacidad ha sufrido una evidente evolución en el último siglo. Desde su identificación con un valor alto de CI en una prueba apropiada, pasando por un acercamiento a los modos de ser de alta capacidad, al considerar las aptitudes diferenciales, hasta imbricar la capacidad o aptitud con el entorno, las oportunidades educativas y un sinfín de variables psicosociales o catalizadores personales y ambientales. De este modo las capacidades, no solo las cognitivas, se entienden en proceso de desarrollo si se dan las circunstancias oportunas, la intervención educativa adecuada. Hoy en día se promueve la importancia de identificar las necesidades educativas de cada escolar para poder ofrecerle la respuesta educativa oportuna, que haga que sus capacidades potenciales se conviertan en rendimiento.

Tenemos que ser capaces de construir dentro de la escuela 'carriles para vehículos rápidos', del mismo modo que tenemos carriles para alumnos más lentos (adaptaciones curriculares, profesores de apoyo, etc.). Los profesores tienen 
que recibir la formación adecuada para que sean capaces de adaptar, al menos, estos cuatro parámetros: profundidad, complejidad, amplitud y velocidad, en planes tan individualizados como sea posible (Tourón, 2012, p. 193).

Este argumento llevado al extremo hará que la escuela sea verdaderamente personalizada y que cada estudiante logre que sus capacidades, pocas o muchas, lleguen a su óptimo desarrollo, haciéndolos verdaderamente competentes.

El establecimiento de una adecuada conceptualización sobre los alumnos más capaces que contemple de manera esencial la dimensión evolutiva, más allá del enfoque del rasgo o atributo estable e inmutable o puntos de corte basados en el CI que determinen 'quien es' y 'quién no es', parece esencial ya que, de otro modo, no se podrá establecer un procedimiento de identificación coherente y, por ello, de intervención.

Las escuelas actuales están muy centradas en la edad como criterio de agrupamiento y en la tarea del profesor como agente principal del proceso de enseñanza. Es preciso que se contemplen de modo más adecuado las diferencias de capacidad, intereses, motivaciones y competencias de los estudiantes, convirtiendo las escuelas en ámbitos de desarrollo del talento, a través de un aprendizaje tan personalizado como sea posible 9 . Hoy en día las tecnologías digitales hacen alcanzable este aparente imposible, haciendo realidad lo que significa una verdadera educación basada en competencias y orientada al dominio ${ }^{10}$.

Es preciso, para ello, que cada escuela evalúe de manera periódica a todos sus escolares en, al menos tres dimensiones: aptitudes, competencias y soft skills o variables psicosociales, catalizadoras del aprendizaje y la productividad. Naturalmente para ello es preciso formación de los profesores, pero actualmente está disponible formación de calidad desde diversas Universidades ${ }^{11}$.

Somos conscientes de que simplemente se ha 'sobrevolado' por un tema tan importante para la justicia y el desarrollo social y que queda por tratar aquí la cuestión, no menor, de la intervención educativa. Podemos remitir al lector a varias listas de contenido sobre estos asuntos ${ }^{12}$.

9 Las cifras oficiales de alumnos de alta capacidad intelectual (otros tipos ni se consideran) en España son preocupantes pues si bien hay diferencias entre comunidades, un $98 \%$ de los alumnos está sin identificar. Puede verse al respecto: https://www.javiertouron.es/la-identificacion-no-es-una-necesidad/

10 Sobre estos dos asuntos se puede ver:

https://www.javiertouron.es/educacion-basada-en-competencias-y-aprendizaje-personalizado/

https://www.javiertouron.es/ensenar-y-aprender-para-el-dominio-o/

11 A modo de ejemplo: https://www.unir.net/educacion/curso-altas-capacidades-intelectuales/549201758643/

12 Desde los siguientes enlaces se puede acceder a listas digitales de contenido sobre los temas no tratados en este artículo:

https://www.pearltrees.com/escuelaunir/estrategias-intervencion/id20231629\#1041

https://www.pearltrees.com/escuelaunir/aceleracion-estrategia/id20231807\#1397

https://www.pearltrees.com/escuelaunir/agrupamiento-rendimiento/id20235527\#1065 


\section{Referencias}

Barnett, L. B. y Corazza, L. (1993). Identification of Mathematical Talent and Programmatic Efforts to Facilitate Development of Talent. European Journal for High Ability, 4, 48-61.

Barnett, L. B. y Gilheany, S. (1996). The CTY Talent Search: International Applicability and practice in Ireland. High Ability Studies, 7(2), 179-191.

Benbow, C. P. (1992). Academic Achievement in Mathematics and Science of Students between Ages 13 and 23: Are there Differences among Students in the Top one Percent of Mathematical Ability? Journal of Educational Psychology, 84(1), 51-61.

Clark, B. (1992): Growing Up Gifted: Developing the Potential of Children at Home and at School (4⿳亠丷a Ed.). New York: Macmillan Publishing Company.

Clynes, T. (2016). How to raise a genius: lessons from a 45-year study of super-smart children. Nature, 537, 152-155. doi:10.1038/537152a. Recuperado de https://www. nature.com/news/how-to-raise-a-genius-lessons-from-a-45-year-study-of-supersmart-children-1.20537

Colangelo, N. y Davis, G. A. (Eds) (2002). Handbook of Gifted Education (3rd Ed.). Boston: Allying \& Bacon.

Colangelo, N., Assouline, S. y Gross, M. (2004). A Nation Deceived: how schools hold back America's brightest students. The Templeton National Report on Acceleration. Iowa City: IA, University of Iowa. Recuperado de: http://www.nationdeceived.org

De Haan, R. y Havighurst, R. (1957). Educating Gifted Children. Chicago: University of Chicago Press.

De la Orden, A. (1975). El agrupamiento de los alumnos: estudio crítico. ICE de la Universidad Complutense: Instituto de Pedagogía del CSIC.

Feldhusen, J. F. y Baska, L. (1985). Identification and Assessment of the Gifted and Talented. En J. F. Feldhusen (Ed.). Toward Excellence in Gifted Education (pp. 70-94). Denver: Love.

Feldhusen, J. F. y Harwan, F. A. (1993). Identification of Gifted and Talented Youth for Educational Programs. En K. A. Heller; F. J. Mönks y A. H. Pasow (Eds.). International Handbook of Research and Development of Giftedness and Talent (pp.271-282). Oxford: Pergamon Press.

Feldman, D. H. (1992). Has There Been a Paradigm Shift in Gifted Education? En N. Colangelo, S. G. Assouline, y D. L. Ambroson (Eds.). Talent Development: Proceedings From the 1991 Henry B. and Jocelin Wallace National Research Symposium on Talent Development (pp.89-94). New York: Trillium Press.

Gagné, F. (2015). De los genes al talento. Revista de Educación, 368, 66-91.

García Hoz, V. (1975). Principios de Pedagogía Sistemática. Madrid: Rialp.

Goldstein, D., Stocking, V. B. y Godfrey, J. J. (1999). What We've Learned from Talent Search Research. En Colangelo, N. y Assouline, S. G. (Ed.). Talent Development III. Proceedings from the 1995 Henry B. and Jocelyn Wallace National Research Symposium on Talent Development (pp. 143-152). Scottsdale, Arizona: Gifted Psychology Press. Guilford, J.P. (1967). The Nature of Human Intelligence. New York: McGraw-Hill. Hagen, E. (1980). Identification of the Gifted. New York: Teachers College Press. 
Heller, K. A., Mönks, F. J., Subotnik, R. y Sternberg, R. (2000). International Handbook on Giftedness and Talent. (2 ${ }^{\text {nd }}$ Ed). Oxford: Pergamon

Hollingworth, L. S. (1929). Gifted Children. Their Nature and Nurture. New York: The Macmillan Company

Jackson, N. E. y Butterfield, E. C. (1986). A Conception of Giftedness Designed to Promote Research. En Sternberg, R. J. y Davidson, J. E. (Eds.). Conceptions of giftedness (pp.151-181). New York: Cambridge University Press

Jiménez, C. (Coord.) (1998). Lecturas de Pedagogía Diferencial. Madrid: Dykinson.

Jiménez, C. y González Galán, A. (2011). Pedagogía Diferencial y atención a la diversidad. Madrid: Editorial Universitaria Ramón Areces.

López López, E., Tourón, J. y González Galán, M. A. (1991). Hacia una Pedagogía de las diferencias individuales: Reflexiones en torno al concepto de Pedagogía Diferencial. Revista Complutense de Ciencias de la Educación, 2(1), 83-92.

Lubinski, D., Benbow, C. P. y Kell, H. J. (2014). Life paths and accomplishments of mathematically precocious males and females four decades later. Psychological Science, 25 (12), 2217-2232. doi:10.1177/0956797614551371

Mönks, F.J. y Pflüger, R. (2005). Gifted Education in 21 European Countries: Inventory and Perspective. Nijmegen: Radbou University. Recuperado de: https://www.pef.uni-lj. si/fileadmin/Datoteke/CRSN/branje/Gifted_Education_in_21_European_Countries_ Inventory_and_Perspective_2005_.pdf

NAGC (2019). A Brief History of Gifted Education. Recuperado de: http://www.nagc. org/resources-publications/resources/gifted-education-us/brief-history-gifted-andtalented-education

Olszewski-Kubilius, P., Subotnik, R. y Worrell, F. C. (2015). Re-pensando las altas capacidades: una aproximación evolutiva. Revista de Educación, 368, 40-65. doi: 10.4438/1988-592X-RE-2015-368-297

Pfeiffer, S. (2012). Current Perspectives on the Identification and Assessment of Gifted Students. Journal of Psychoeducational Assessment, 30(1), 3-9. doi: $10.1177 / 0734282911428192$.

Pfeiffer, S. (2017). Identificación y evaluación del alumnado con altas capacidades: Una guía práctica. La Rioja: UNIR Editorial.

Piirto, J. (1999). Talented Children and Adults. Their Development and Education (2 ${ }^{\mathrm{a}}$ Ed.). New Jersey: Prentice-Hall.

Reyero, M. y Tourón, J. (2000). Reflexiones en torno al concepto de superdotación: evolución de un paradigma. Revista Española de Pedagogía, 215, 7-38.

Richert, E. S. (1991). Rampant Problems and Promising Practices in Identification. En N. Colangelo y G. A. Davis (Eds.). Handbook of Gifted Education (pp. 75-88). Boston: Allyn and Bacon.

Richert, E. S.; Alvino, J. J. y McDonnel, R. C. (1982). National Report on Identification: Assessment and Recommendations for Comprehensive Identification of Gifted and Talented Youth. New Jersey: A Product of the Educational Information and Resource Center.

Shavinina, L. (Ed.) (2009). International Handbook on Giftedness. The Netherlands: Springer Verlag.

Stanley, J. C. y Benbow, C. P. (1982). Educating Mathematically Precocious Youths: Twelve Policy Recommendations. Educational Researcher, 11, 4-9. 
Stanley, J. C. y Benbow, C. P. (1983). SMPY's First Decade: Ten Years of Posing Problems and Solving Them. Journal of Special Education, 17(1), 11-25.

Stanley, J. C. y Benbow, C. P. (1986). Youths Who Reason Exceptionally Well Mathematically. En R. J. Sternberg, y J.E. Davidson, J. E. (Eds.). Conceptions of Giftedness (pp. 20-37). New York: Cambridge University Press.

Subotnik, R. F., Olszewski-Kubilius, P., y Worrell, F. C. (2011). Rethinking giftedness and gifted education: A proposed direction forward based on psychological science. Psychological Science in the Public Interest, 12, 3-54. doi:10.1177/1529100611418056.

Tannembaun, A. J. (1983). Gifted Children: Psychological and Educational Perspectives. New York: Macmillan.

Tourón, J. (2004), De la superdotación al talento: evolución de un paradigma. En Jiménez, C. Pedagogía Diferencial. Diversidad y Equidad. Madrid: Pearson. pp. 369-400.

Tourón, J. (2012). ¿Superdotación o alta capacidad? Entrada de blog: https://www. javiertouron.es/superdotacion-o-alta-capacidad/

Tourón, J. y Reyero, M. (2002). Identificación y diagnóstico de alumnos de alta capacidad. Bordón, 54 (2), 311-338

Tourón, J. y Reyero, M. (2001). The Talent Search Model and acceleration: What the research tells us? Educating Able Children, 5(2), 18-35.

Tourón, J. y Reyero, M. (2002). The Implementation of the Talent Search Concept in Spain. NATO Science Series, 47, 63-76.

Tourón, J. (2011). The Center for Talented Youth Identification Model: A Review of the Literature. Talent Development and Excellence, 3(2), 187-202.

Tourón, J. (2012). El agrupamiento por capacidad en el caso de los alumnos más capaces. En M. Castro Morera (Coord.). Elogio a la Pedagogía Científica. Un Liber Amicorum para Arturo de la Orden Hoz (pp. 187-230). Madrid: Autor Editor.

Tourón, J. y Tourón, M. (2016). Identification of Verbal and Mathematical Talent: The Relevance of 'Out of Level' Measurement. Anales de Psicología, 32(3), 638-651.

Tourón, J., Réparaz, Ch. y Peralta, F. (2006). Las nominaciones de los profesores en la identificación de alumnos de alta capacidad intelectual. Sobredotação, 7, 7-30.

Tourón, J.; Peralta, F. y Repáraz, C. (1998). La Superdotación Intelectual: Modelos, Identificación y Estrategias Educativas. Pamplona: EUNSA.

Tourón, J., Tourón, M. y Silvero, M. (2005). The Center for Talented Youth Spain: An Initiative to Serve Highly Able Students. High Ability Studies, 16(1), 121-135.

Treffinger, D. J. (1991). Future Goals and Directions. En Colangelo N. y Davis, G. A. (Eds.) Handbook of Gifted Education. Boston: Allyn and Bacon.

Treffinger, D. J. y Feldhusen, J. F. (1996). Talent Recognition and Development: Successor to Gifted Education. Journal for the Education of the Gifted, 19(2), 181-193.

Vázquez Gómez, G. (1981). Apuntes biográficos de la ciencia. Revista Española de Pedagogía, 39(153), 9-36.

Fecha de recepción: 23 de septiembre de 2019.

Fecha de revisión: 14 de octubre de 2019.

Fecha de aceptación: 17 de octubre de 2019. 